\title{
EFFECT OF IN-BAND GROUP DELAY RIPPLE ON WDM FILTER PERFORMANCE
}

\author{
Christos Riziotis ' and Michalis N. Zervas ${ }^{1,2}$ \\ (1) Optoelectronics Research Centre, University of Southampton, Southampton, SOl7 1BJ, 'UK \\ (2) Southampton Photonics Inc., Chilworth Science Park, Southampton S016 7NS,UK.
}

The effects of time-delay-ripple period and amplitude in $25 \mathrm{GHz}$ and JOOGHzfilters for operation at JOGbls and 40Gbls, respectively, are studied. It is shown that maximum transmission penalties occur at time-delay periods of- $75 \%$ of the-20dB data bandwidth.

\section{Introduction}

WDM optical systems are continuously migrating towards shorter channel spacings and higher bit rates in order to use the available bandwidth most efficiently. Various combinations of channel spacings ranging from $25 \mathrm{GHz}$ to $100 \mathrm{GHz}$, and bit rates from $10 \mathrm{~Gb} / \mathrm{s}$ to $40 \mathrm{~Gb} / \mathrm{s}$ are currently explored. High performance optical filters are proving to be central to the successful deployment of high-speed optical systems. High bandwidth efficiency sets quite stringent requirements to WDM filter characteristics and any imperfections in their phase and/or amplitude response become critical.

WDM filters based on fibre-grating [1] and thin-film technologies are known to show in-band time delay ripples due to design and manufacturing compromises. The thorough study and understanding of the impact of timedelay ripple period and amplitude on the transmission penalties associated with the use of these filters is of paramount importance. This will help in defining design tolerances and trade-offs necessary for the development of highly efficient filters. In-band monotonic dispersion variation is known to limit the efficiency of grating filters [2]. Periodic in-band time delay ripple has been shown to affect the performance of chirped fibre grating used as dispersion compensators [3].

In this paper, we examine the effects of time-delayripple period and amplitude in $25 \mathrm{GHz}$ and $100 \mathrm{GHz}$ filters for operation at $10 \mathrm{~Gb} / \mathrm{s}$ and $40 \mathrm{~Gb} / \mathrm{s} \mathrm{NRZ} \mathrm{data,} \mathrm{respectively.}$ Using simple analysis, we also demonstrate that maximum transmission penalties are associated with periods that correspond to peak average time-delay ripple (averaged over the data bandwidth). In all cases, this period is found to be about $75 \%$ of the data bandwidth.

\section{Time Delay Ripple}

The filter in-band time delay ripple is defined as:

$$
\Delta \tau(\lambda)=a_{n} \cdot\left(\lambda-\lambda_{o}\right)^{2 n}+a_{o} \cos \left(\frac{2 \pi}{p_{o}} \lambda+\theta\right)
$$

where $\alpha_{0}$ and $p_{0}$ are the time delay ripple amplitude, the period, respectively. $\theta$ determines the relative position of the time delay peaks with respect to the filter centre wavelength. The first term in Eqn. (1) gives the monotonic time-delay increase towards the band-edges observed in most of the used optical filters. Figure 1, shows these three representative cases with $p_{0}=100 \mathrm{pm}$ and $\theta=0, \pi / 2$, and $\pi$.

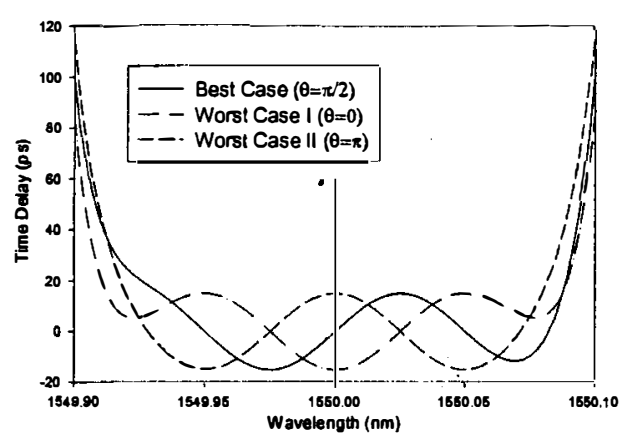

Figure 1: In-band time-delay variation with $p_{0}=100 \mathrm{pm}$ and $\theta=0, \pi / 2, \pi$.

\section{EOP Calculations}

Using system simulations in an Intensity Modulation/Direct Detection (IM/DD) transmission system according to SONET standards, we estimate the eye-opening penalty (EOP) arising from the ripple effects. Here a perfectly rectangular filter with bandwidth $25 \mathrm{GHz}$ is employed and a signal modulated at $\mathrm{NRZ}$ format at $10 \mathrm{~Gb} / \mathrm{s}$ is transmitted through the filter. Then the recovered signal is characterised by the EOP.

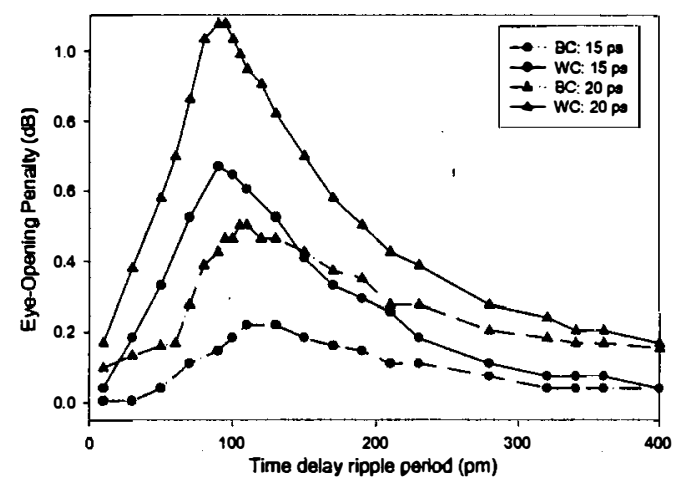

Figure 2: EOP for $10 \mathrm{~Gb} / \mathrm{s} \mathrm{NRZ}$ data and $25 \mathrm{GHz}$ filter.

Figure 2 gives the EOP as a function of the ripple periodicity for two different ripple amplitudes. The time delay pattern which corresponds to $\theta= \pm \pi / 2$ exhibits the lowest EOP (best case), while the cases with $\theta=0, \pi$ give the most degraded performance (worst case). For all the other $\theta \mathrm{s}$, the EOP lies between these two extremes. For both amplitudes, a resonance behaviour is observed with the worst-case EOP peaking for a resonance period of $\sim 90 \mathrm{pm}$. 


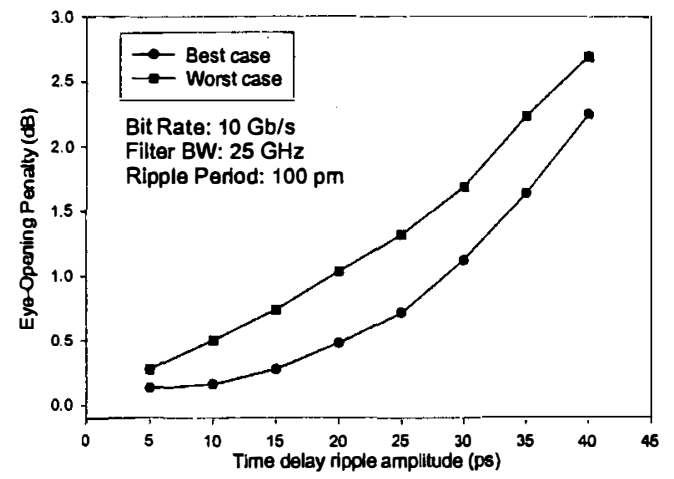

Figure 3: Effect of ripple amplitude at $10 \mathrm{~Gb} / \mathrm{s} \mathrm{NRZ} \mathrm{data.}$

For any ripple period, the EOP increases monotonically with the ripple amplitude. Figure 3 shows the calculated EOPs for a ripple period of $100 \mathrm{pm}$. It is shown that for ripple amplitudes smaller that $\sim 25 \mathrm{ps}$, the worst-case EOP increases quasi-linearly with the amplitude. For negligible additional transmission penalty (EOP $\leq 0.3 \mathrm{~dB})$, in the worstcase scenario (i.e., at resonance period $\approx 90 \mathrm{pm}$ ) it is found that the optical filter should exhibit time-delay ripple amplitude smaller than $\sim 3$ ps. For periods away from the resonance one, larger time-delay ripple amplitudes can be tolerated.

We also examined the effect of the time delay ripples in the case of NRZ data transmission at $40 \mathrm{~Gb} / \mathrm{s}$ through a $100 \mathrm{GHz}$ rectangular filter. Figure 4 shows the worst and best cases for ripple amplitude of 5ps. The worst-case EOP shows the same resonance behaviour peaking at a period of $\sim 350 \mathrm{pm}$. This resonance period is roughly four times the corresponding value at $10 \mathrm{~Gb} / \mathrm{s}$. However, we should stress that, compared to $10 \mathrm{~Gb} / \mathrm{s}$ case (see Fig. 2), the same maximum worst-case EOP $(\sim 1.4 \mathrm{~dB})$ is achieved with only $1 / 4$ of the ripple amplitude. Higher bit rates put much more stringent requirements on filter characteristics.

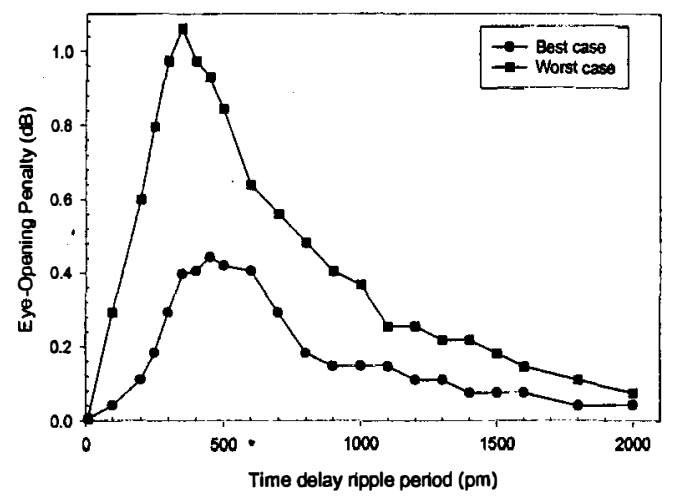

Figure 4: EOP for $40 \mathrm{~Gb} / \mathrm{s} \mathrm{NRZ} \mathrm{data} \mathrm{and} 100 \mathrm{GHz}$ filter.

\section{Time-Delay Ripple Figure of Merit}

In order to understand the resonant dependence of the signal degradation penalties on time-delay ripple period better, we introduce the following parameter that gives a measure of the average time-delay ripple size, namely:

$$
\begin{aligned}
& \Delta \tau_{\text {ripple }}=\sqrt{\frac{1}{B W}} \int_{-B W / 2}^{+B W / 2} \Delta \tau^{2}(\lambda) d \lambda-\Delta \tau_{\text {average }} \\
& \text { where } \quad \Delta \tau_{\text {average }}=\frac{1}{B W} \int_{-B W / 2}^{+B W / 2} \Delta \tau(\lambda) d \lambda
\end{aligned}
$$

and $\mathrm{BW}$ is the data bandwidth. By substituting (1) into (2), we obtain:

$$
\begin{aligned}
& \Delta \tau_{\text {ripple }}=a_{o}\left\{\sqrt{\frac{1}{2}\left[1+\cos (2 \theta) \frac{\sin (2 x)}{2 x}\right]}-|\cos (\theta)| \frac{\sin (x)}{x}\right\} \\
& x=\pi B W / p_{0} .
\end{aligned}
$$

In Figure 5, the average ripple is plotted as a function of the ratio of ripple period over the data bandwidth $\left(p_{0} / \mathrm{BW}\right)$, for the worst case $(\theta=0, \pi)$ and $\alpha_{0}=15 \mathrm{ps}$. In this case, the average time-delay ripple size exhibits a resonant peak for $(p / \mathrm{BW}) \approx 0.75 \mathrm{BW}$. For NRZ data at $10 \mathrm{~Gb} / \mathrm{s}$, the $-20 \mathrm{~dB}$ bandwidth is about $B W_{-20}=15 \mathrm{GHz}(\equiv 120 \mathrm{pm})$ that results in a resonant period $p_{\mathrm{r}} \sim 90 \mathrm{pm}$. At $40 \mathrm{~Gb} / \mathrm{s}$, the $-20 \mathrm{~dB}$ data bandwidth is about $B W_{-20}=60 \mathrm{GHz}(\equiv 480 \mathrm{pm})$ that results in a resonant period $p_{\mathrm{r}} \approx 360 \mathrm{pm}$. These resonance periods are in excellent agreement with the ones obtained from the EOP results (see Figs (2), (4)).

Periodic in-band time delay results of course in periodic in-band dispersion, which can be easily calculated analytically (in a similar manner to Eqn (3)). The inset in Figure 5 plots the corresponding average dispersion ripple size. Interestingly the dispersion shows a monotonically decreasing behaviour and cannot describe the resonance characteristics of the transmission degradation.

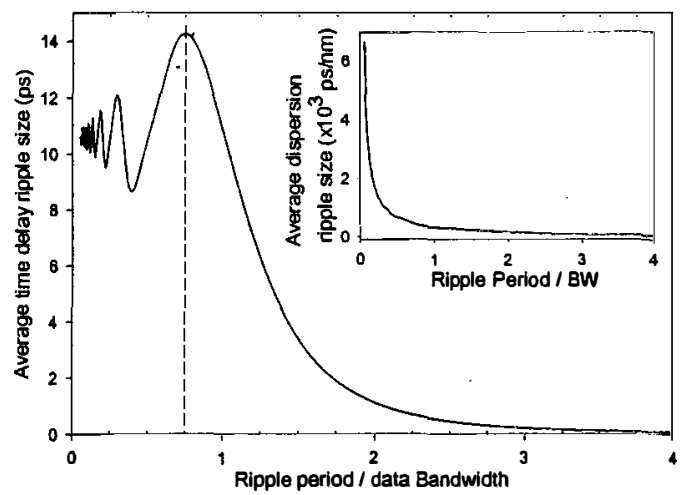

Fig. 5: Average time-delay and dispersion ripple (worst case).

\section{Conclusions}

In conclusion, we have studied the additional EOP due inband periodic time delay variation of optical filters showing a distinct resonance characteristics with the ripple period. The worst-case resonance ripple period $\left(p_{\mathrm{r}}\right)$ scales with the $-20 \mathrm{~dB}$ data bandwidth $\left(B W_{-20}\right)$. Actually $p_{\mathrm{r}} \approx 0.75$ $B W_{-20}$. The ripple amplitude $\left(\alpha_{0}\right)$ required to achieve certain worst-case maximum EOP, is inversely proportional to $p_{\mathrm{r}}\left(\alpha_{0} \propto 1 / p_{\mathrm{r}}\right)$. For periods away from $p_{\mathrm{r}}$, larger ripple amplitudes can be tolerated.

Finally, in specifying the optical filter performance, average time-delay ripple over the bandwidth data and ripple period should be considered. Average dispersion or dispersion ripple over the filter bandwidth seems to have no direct correlation with the observed filter-induced signal degradation.

\section{References}

/1/ M. Ibsen, et al, paper MoDl, OFC' 2001.

/2/ G. Nykolak, IEEE Photon. Technol. Lett., Vol. 10, No. 9, pp. 1319-1321 (1998).

/3/ K. Ennser, et. al., IEEE Photon. Technol. Lett., Vol. 10, No 10, pp. 1476-1478 (1998). 